\title{
¿Qué ciencia se presenta en los libros de texto de Educación Secundaria?
}

\section{Which type of science is presented in Secondary Education textbooks?}

\author{
Margarita Minerva Ibáñez Ibáñez, María del Carmen Romero López, María del Pilar Jiménez Tejada \\ Departamento de Didáctica de las Ciencias Experimentales. Universidad de Granada, España. \\ margaritaibanezibanez@gmail.com, romero@ugr.es, pjtejada@ugr.es
}

RESUMEN • Este trabajo muestra un análisis de los temas que introducen la ciencia en los libros de Biología y Geología y Física y Química de Educación Secundaria. El objetivo es caracterizar la visión de la "naturaleza de la ciencia» que estos presentan, comprobar si se diferencia ciencia de pseudociencia y estudiar la presencia de ciertos conceptos. Para ello se han analizado tanto los contenidos teóricos como las actividades. El análisis revela que varios libros carecen de un tema introductorio. En los que sí lo tienen, se observa que suele centrarse en el método científico y presta una atención limitada a la evolución de la ciencia, los modelos científicos u otros conceptos relevantes. La imagen de ciencia que se promueve, si bien incluye aspectos positivos, no es aún, por lo general, completamente correcta. Se aprecia, por tanto, una falta de reflexión sobre qué es la ciencia y cómo se construye.

PALABRAS CLAVE: Educación secundaria; Libros de texto; Naturaleza de la ciencia; Pseudociencia; Metodología científica.

\begin{abstract}
This paper presents a study based on an analysis of introductory lessons to biology and geology and to physics and chemistry in Secondary Education textbooks. The aims of this study are to characterize the view on the "nature of science» that is transmitted, to determine whether science is differentiated from pseudoscience and to detect whether certain concepts appear, by examining both the theoretical content of the textbooks and the recommended activities. The analysis reveals a lack of an introductory lesson in some books. Where such lesson is included, the text usually focuses on scientific method, paying little attention to the evolution of science, scientific models or other significant concepts. The image of science that is promoted, although it has positive aspects, is often deficient. In short, there is a lack of reflection on what science is and how it is constructed.
\end{abstract}

KEYWORDS: Secondary education; Textbooks; Nature of science; Pseudoscience; Scientific methodology.

Recepción: mayo 2018 • Aceptación: diciembre 2018 • Publicación: noviembre 2019 


\section{INTRODUCCIÓN}

La importancia de enseñar conocimientos científicos nunca ha estado en duda y durante años se ha centrado en la transmisión del conocimiento acumulado. Sin embargo, desde los años sesenta se viene manifestando el deseo de incluir también en los currículos de ciencias el conocimiento sobre la propia ciencia (Lederman, 2007; McComas, Clough y Almazroa, 1998). Qué es la ciencia, cómo funciona, cómo trabajan los científicos y cómo se relacionan ciencia y sociedad es lo que hoy conocemos como naturaleza de la ciencia (NdC) (Clough, 2006; McComas et al., 1998) y que da respuesta a estos interrogantes.

La concepción sobre la ciencia ha ido variando a lo largo de los años, moviéndose entre el positivismo y el relativismo, pero estamos de acuerdo con González-Galli (2010) en que comprender la ciencia implica alcanzar una visión intermedia entre ambos. Y si bien la comunidad científica ha llegado a cierto acuerdo, el cambio de mentalidad en toda la población lleva más tiempo, por lo que aún son frecuentes concepciones deformadas de la ciencia que están muy extendidas (Afonso y Gilbert, 2010; Koskal, Cakiroglu y Geban, 2013; Vílchez-González, Carrillo-Rosúa, Rodríguez-Sabiote y JiménezTejada, 2015), y que con frecuencia son mostradas en los libros de texto (McComas, 1998). Ejemplo de ello pueden ser el apelativo el método científico, donde el artículo el lleva a la idea de que es exclusivo (Woodcock, 2014); la visión de la experimentación como una etapa crucial, y las relaciones hipótesisley-teoría, en las que las hipótesis se convierten en leyes (no siempre es así) y las leyes en teorías o viceversa (McComas, 1998; McComas, 2003; Woodcock, 2014).

Por esto, un motivo importante para incluir la $\mathrm{NdC}$ en la enseñanza sería cambiar las concepciones erróneas que la población posee sobre ella. Lederman (2007) indica que comprender la ciencia como disciplina pasa por entender también la $\mathrm{NdC}$, pero además el profesorado de ciencias tiene en sus manos el deber moral de difundir cómo se ha construido el pensamiento científico. Para educar en $\mathrm{NdC}$ debemos considerar no solo los aspectos en los que existe acuerdo, sino también ampliar con otros como la relación recíproca entre sociedad y ciencia y tecnología o la sociología interna de la ciencia (Acevedo-Díaz y García-Carmona, 2016).

Varias investigaciones sugieren que se obtienen mejores resultados en la enseñanza de la $\mathrm{NdC}$ cuando esta se lleva a cabo bajo un enfoque explícito y reflexivo (Acevedo, 2009; Khishfe y Abd-El-Khalick, 2002; Koskal et al., 2013; Lederman y Lederman, 2014). Partiendo de ahí, trabajar los modelos científicos en el aula, así como analizar la evolución de la ciencia, pueden ser procedimientos excelentes para mostrar cómo funciona esta. De hecho, el uso de la historia de la ciencia como recurso para enseñar la NdC ha sido ya propuesto por varios autores (Acevedo-Diaz, García-Carmona y Aragón, 2017; Allchin, 2012; Solbes y Traver, 2001). Y la necesidad de hacer un uso reflexivo de los modelos, así como su potencial utilidad para abordar la NdC, ha sido puesta de manifiesto por Domènech-Casal (2015), Galagovski y Adúriz-Bravo (2001) y Oliva, Aragón-Méndez, Jiménez-Tenorio y Aragón-Núñez (2016).

La investigación sobre la imagen de la ciencia que aparece en los libros de texto ha sido objeto de numerosos análisis, encontrándose que la ciencia suele presentarse como una acumulación de hechos donde no se hace referencia ni a los debates o dificultades que se pudieron presentar durante su desarrollo, ni al contexto histórico o social en el que tuvieron lugar (Occelli y Valeiras, 2013; SolazPortolés, 2010). Occelli y Valeiras (2013) lo resumen como un énfasis en el producto, pero no en el proceso. Además, la actividad científica suele presentarse con un carácter individualista, en el que diferentes científicos llegaron a distintos descubrimientos mediante un método inductivo o experimental. Los textos olvidan destacar el componente creativo e imaginativo, mostrando, en palabras de SolazPortolés (2010), una imagen algorítmica de la metodología científica, y omitiendo la importancia de la comunidad científica para validar el conocimiento.

Un concepto íntimamente relacionado con el de ciencia, aunque opuesto, es el de pseudociencia. Algunas de sus fortalezas radican en su apariencia de ciencia -reclamo de autoridad del que se apro- 
vechan-, en sus atractivas afirmaciones y en su rápida difusión desde los medios de comunicación (Alonso-Marcos y Sergi-Cortiñas, 2014). Ante esta situación, se hace patente que la vía para reducir las creencias pseudocientíficas debería encaminarse directamente hacia la crítica a la pseudociencia, la cual, además, puede aprovecharse también para comprender mejor la ciencia y su naturaleza (Good, 2012). Para ello la argumentación y la discusión entre compañeros se han mostrado como herramientas eficaces (Çetinkaya, Turgut y Duru, 2015; Tsai, Lin, Shih y Wu, 2015; Yu y Yore, 2013). Finalmente, es también necesario recordar que el papel de la mujer en la ciencia sigue estando poco valorado, que los estereotipos de género comienzan a aparecer a edades tempranas y que, según mostró el proyecto internacional ROSE (Relevance of Science Education), las chicas tienen una actitud menos positiva que los chicos respecto a la ciencia escolar (Bian, Leslie y Cimpian, 2017; Sjøberg y Schreiner, 2010; Vázquez y Manassero, 2009). Afortunadamente la legislación vigente no es ajena a esta necesidad y marca como elemento transversal el fomento de la igualdad, y más específicamente dice: «... el reconocimiento de la contribución de ambos sexos al desarrollo de nuestra sociedad y al conocimiento acumulado...»(BOJA n. ${ }^{\circ}$ 144, de 28/07/2016, p. 110).

\section{OBJETIVOS}

Es más que sabido que los libros de texto son una herramienta muy usada en el aula (Occelli y Valeiras, 2013), por lo que resulta interesante conocer cómo se trata la NdC en ellos. En este trabajo se presenta un análisis de libros de texto de Educación Secundaria que tiene por objetivo caracterizar la visión de la naturaleza de la ciencia que se promueve en los temas de introducción a la ciencia, tanto en los contenidos teóricos como en las actividades.

Para lograrlo se plantean los siguientes objetivos específicos:

- Comprobar si las descripciones de ciencia y de metodología científica que aparecen en ellos son acordes con la visión actual de la $\mathrm{NdC}$.

- Averiguar si se diferencia ciencia de pseudociencia.

- Corroborar la presencia de modelos científicos.

- Estudiar si está presente la evolución de la ciencia y si aparece la contribución de la mujer.

\section{METODOLOGÍA}

Se han analizado 31 libros (anexo 1) de Educación Secundaria Obligatoria (ESO) pertenecientes a la Ley Orgánica de Mejora de la Calidad Educativa (LOMCE): 15 de Biología y Geología (BG) y 16 de Física y Química (FQ).

Para la revisión se diseñó un protocolo validado por profesores del Departamento de Didáctica de las Ciencias Experimentales de la Universidad de Granada (anexo 2).

Se analizó la presencia, localización, actividades y ejemplos de los siguientes aspectos:

- Existencia de un bloque de contenidos dedicado a la introducción a la ciencia

- Definición de ciencia

- Enfrentamiento pseudociencia vs. ciencia

- Descripción de la metodología científica

- Presencia de modelo/s científico/s

- Desarrollo de la evolución de la ciencia

- Contribución de la mujer a la ciencia

Los resultados se analizaron mediante una metodología cualitativa con registro numérico. 


\section{RESULTADOS Y DISCUSIÓN}

\section{Existencia de un bloque de contenidos dedicado a la introducción a la ciencia}

Del total de libros analizados, en el $35 \%$ de ellos no existe un bloque de introducción a la ciencia. Sin embargo, en 9 hay propuestas relacionadas con la investigación y su metodología. Pueden ser proyectos de investigación guiados, que deba desarrollar el alumno (6 libros), o ejemplos desarrollados completamente por el libro (3 libros) (figura 1). Posiblemente con estos intenten cumplir con el último bloque de contenidos del currículo oficial de BG: «B7. Proyecto de investigación» en $1 .^{\circ}$ y $3 .^{\circ}$ de la ESO y «B4. Proyecto de investigación» en 4. (MECD, 2014, pp. 210 y 213). En tal caso, los proyectos que deba desarrollar el alumno se ajustarían mejor a los criterios de evaluación presentados en dicho apartado del currículo, ya que estos están vinculados al «hacer». No obstante, los ejemplos del libro son muy completos e ilustrativos, pudiendo ser de ayuda en caso de que no se pudieran realizar los proyectos de investigación.

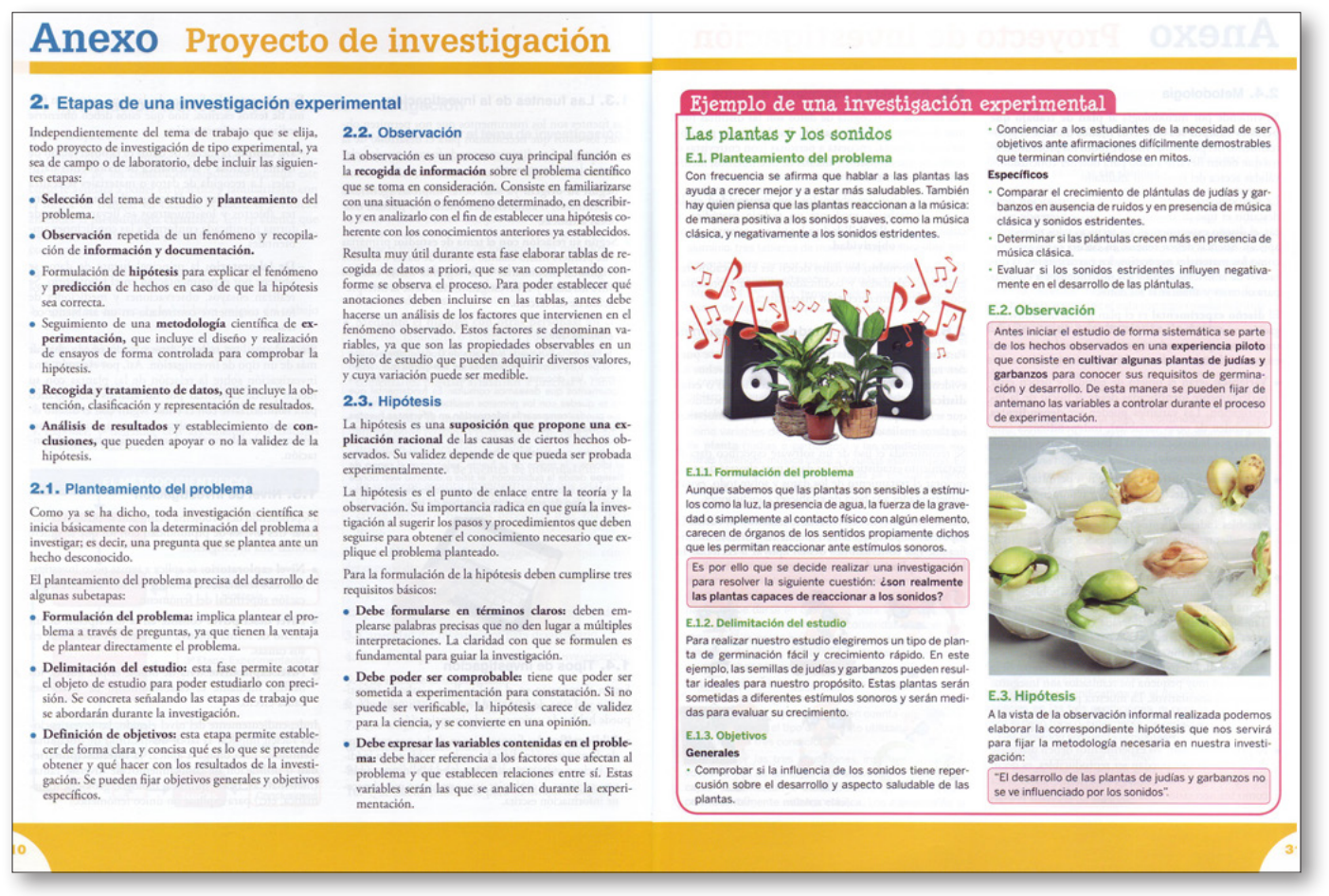

Fig. 1. Proyecto de investigación desarrollado por el libro. BG, 1. ESO, 2016, Algaida

Así, la muestra final se redujo a los 20 libros que sí contenían dicho bloque. No obstante, solo en 5 de ellos conforma un tema completo, mientras que en el resto forma parte de un tema (junto a contenidos relacionados con la magnitud y la medida), y en uno aparece como apéndice final. Este último caso pertenece al libro de BG de $10^{\circ}$ de la ESO de SM, y consiste en una serie de cómics históricos titulados Locos por la ciencia. Estos resultados no concuerdan con lo que especifica el Real Decreto 1105/2014, de 26 de diciembre, por el que se establece el currículo básico de la Educación Secundaria Obligatoria y del Bachillerato (MECD, 2014). En él se fija como primer bloque de contenidos «la metodología o actividad científica» en todos los cursos de la ESO, tanto en FQ como en BG (excepto para 4. ${ }^{\circ}$ de la ESO de BG). Aunque en el Real Decreto aparecen juntos los contenidos de BG de $1 .^{\circ}$ y 3. ${ }^{\circ}$, y los de FQ de $2 .^{\circ}$ y 3. ${ }^{\circ}$, la Orden de 14 de julio de 2016 (BOJA n. ${ }^{\circ}$ 144, de 28/07/2016) mantiene 
en todos los casos este primer bloque. Estas carencias no supondrían un problema si se solventasen en el resto de bloques de contenidos con la guía del profesor.

Los contenidos analizados no reciben la misma atención en las dos asignaturas (tabla 1). Posiblemente algunas editoriales han decidido no repetir contenidos en ambas, optando por ponerlos en FQ. Quizá esta decisión sea una reminiscencia de las dificultades que tuvo la biología para ser considerada como otra disciplina científica (Bermúdez, 2015). Además, hay que contemplar la posibilidad de que en un centro no se mantenga la misma editorial para ambas asignaturas, por lo que sería posible que el estudiante nunca tuviera contacto con dichos contenidos.

Tabla 1.

Distribución de los contenidos de introducción a la ciencia por asignatura y curso

\begin{tabular}{|c|c|c|c|c|c|}
\hline \multirow{2}{*}{ Asignatura } & \multirow{2}{*}{ Curso } & \multirow{2}{*}{$N .^{\circ}$ de libros } & \multicolumn{3}{|c|}{ N. ${ }^{\circ}$ desglosado según tipo de sección } \\
\cline { 4 - 6 } & & & Tema completo & Parte de un tema & Apéndice \\
\hline \multirow{3}{*}{ BG } & $1 .^{\circ}$ & 4 & 3 & 0 & 1 \\
\cline { 2 - 6 } & $3 .^{\circ}$ & 1 & 1 & 0 & 0 \\
\hline \multirow{3}{*}{ FQ } & $2 .^{\circ}$ & 2 & 1 & 1 & 0 \\
\cline { 2 - 6 } & $3 .^{\circ}$ & 11 & 0 & 11 & 0 \\
\cline { 2 - 6 } & $4 .^{\circ}$ & 2 & 0 & 2 & 0 \\
\hline
\end{tabular}

Han llamado nuestra atención dos editoriales: Anaya, donde el tema aparece sin numerar y con las páginas de color diferente, y Edelvives de BG de 1. ${ }^{\circ}$ de la ESO, donde aparece como «Unidad cero». Quizá esa diferencia de formato se deba a que son contenidos que pueden consultarse a lo largo del curso. Aunque sería interesante conocer cómo perciben este hecho los docentes, pues tal vez podrían obviarlo, impartiendo el temario a partir de la unidad 1.

\section{La ciencia y su naturaleza}

Aunque definir ciencia resulta complejo, utilizar una descripción o nombrar algunas de sus características facilitaría su comprensión e introducción. En nuestro estudio hemos considerado que se hacía una descripción de la ciencia, aun cuando no se mencionara expresamente; por ejemplo: «El conocimiento científico surge de la necesidad de dar respuesta...» (FQ, 2. de la ESO, 2016, Anaya). El número de libros que cuenta con esta información es mejorable.

Mientras que en el $30 \%$ de los libros aparece tanto una definición como una descripción, en un $25 \%(\mathrm{n}=20)$ no se da ninguna. Esta ausencia ha llamado nuestra atención, pues se trata de un tema de introducción a la ciencia. Cuando aparece se sitúa normalmente en el cuerpo del texto (tabla 2) y, a veces, de manera simultánea en otras localizaciones. Quizá sería conveniente que, aun estando en el cuerpo del texto, se encuadre dicha información para resaltar su interés. En dos casos aparece solo en la portada del tema y en otro se sitúa en un recuadro de los márgenes y como contraposición de pseudociencia. 
Tabla 2 .

Porcentaje de libros según localización del concepto de ciencia $(n=20)$

\begin{tabular}{|l|c|c|}
\hline \multicolumn{1}{|c|}{ Localización en el libro } & Definición \% & Descripción \% \\
\hline En el cuerpo del texto y encuadrada & 20 & 0 \\
\hline En el cuerpo del texto sin encuadrar & 10 & 55 \\
\hline Otras & 0 & 15 \\
\hline
\end{tabular}

De los 15 libros que definen o describen ciencia, en 13 se alude al hecho de explicar, conocer o estudiar los fenómenos naturales y/o el mundo que nos rodea (figuras 2 y 3), y en 9 se menciona la necesidad de utilizar un método de trabajo adecuado para llegar a esas respuestas. Dicha forma de trabajo, según se puede extraer de los textos, caracterizaría y diferenciaría el conocimiento científico de aquello que no lo es. Los libros de FQ de 2..$^{\circ}$ y $30^{\circ}$ de la ESO de Anaya describen el conocimiento científico como «una construcción del ser humano». Esta descripción está en consonancia con la visión actual de la $\mathrm{NdC}$, pues lleva asociada la idea de que la ciencia no puede ser completamente objetiva.

La ciencia es un conjunto de conocimientos sobre el mundo obtenidos mediante la observación, la experimentación y el razonamiento. El objetivo de la ciencia es conocer la estructura del universo y las leyes que rigen los fenómenos naturales.

Fig. 2. Definición de ciencia. FQ, 3. ESO, 2016, Algaida

El conocimiento científico surge de la necesidad de dar respuesta a un
problema basado en hechos, o fenómenos, a los que se quiere dar expli-
cación.
Pero esta respuesta no se puede obtener de cualquier manera, sino que
se ha de llegar a ella de modo que se asegure que las conclusiones obte-
nidos sean acordes con la realidad. De no ser así, no sería conocimiento
científico.
La figura inferior muestra un esquema simplificado de estos métodos de
trabajo, que se reúnen bajo la expresión «método científico».

Fig. 3. Descripción de conocimiento científico. FQ, 2. ESO, 2016, Anaya

Las características de la $\mathrm{NdC}$ que se han considerado en este apartado siguen las descripciones de Lederman (citado en Vázquez y Manassero, 2012), McComas (2005, citado en Vázquez y Manassero, 2012) y Park, Nielsen y Woodruff (2014). Con respecto a estas, se ha observado que un libro contradice la idea de que el conocimiento científico es parcialmente subjetivo, y el $15 \%(n=20)$ contradice la idea de que el método científico no es exclusivo, lo que podría llevar al estudiante a creer que solo existe un método científico y, normalmente, asociado en exclusiva a la experimentación en laboratorio. Hay que destacar la existencia de pequeñas referencias en los libros de texto que apoyan la visión actual de la $\mathrm{NdC}$ (tabla 3), aunque ante la baja aparición de varias de estas características y las matizaciones que se harán más adelante sobre los libros que muestran la diversidad metodológica, el contenido a este respecto está incompleto. 
Tabla 3.

Porcentaje de libros que presentan las características de la $\mathrm{NdC}$

\begin{tabular}{|c|c|c|}
\hline Características de la $N d C$ & $\begin{array}{l}\text { Libros que la incluyen } \\
\quad(n=20)\end{array}$ & Ejemplos \\
\hline Basada en evidencia empírica & $25 \%$ & $\begin{array}{l}\text { «Se basa en pruebas; nunca se puede basar en creencias, intui- } \\
\text { ciones o suposiciones» (FQ, 2. }{ }^{\circ} \text { ESO, 2016, Anaya) }\end{array}$ \\
\hline Diversidad metodológica & $50 \%$ & $\begin{array}{l}\text { "Cada problema necesita ser tratado de una manera distinta, y } \\
\text { no existe un método previamente establecido para resolverlo" } \\
\text { (FQ, 4. }{ }^{\circ} \text { ESO, 2016, Edelvives) }\end{array}$ \\
\hline $\begin{array}{l}\text { Tentativa. Provisional } \\
\text { y autocorregible }\end{array}$ & $65 \%$ & $\begin{array}{l}\text { «La ciencia está en continuo avance y construcción... Basta } \\
\text { con que se descubra u observe algo que contradiga la hipótesis } \\
\text { aceptada para que esta deba ser reconsiderada o rechazada» } \\
\text { (FQ, 3. ESO, 2016, Bruño) }\end{array}$ \\
\hline Parcialmente subjetiva. Creativa & $45 \%$ & $\begin{array}{l}\text { «La observación de la naturaleza no es objetiva, ya que depen- } \\
\text { de de las teorías que conozca y sostenga el observador» (FQ, } \\
3 .^{\circ} \text { ESO, 2015, SM) }\end{array}$ \\
\hline $\begin{array}{l}\text { Afectada por el marco } \\
\text { sociocultural }\end{array}$ & $15 \%$ & $\begin{array}{l}\text { "La ciencia no es neutra... "solo el } 10 \% \text { de la investigación } \\
\text { médica se dedica a los males que causan el } 90 \% \text { de las muer- } \\
\text { tes”. Desde } 1976 \text { se conoce la gravedad del Ébola, pero, al ser } \\
\text { sus víctimas...» (FQ, 3. ESO, 2015, SM) }\end{array}$ \\
\hline Con límites & $15 \%$ & $\begin{array}{l}\text { «La ciencia solo puede estudiar aquello que puede medir y } \\
\text { observar... Pero no puede establecer juicios de valor subjeti- } \\
\text { vos...» (BG, 3. } .^{\circ} \text { ESO, 2015, Bruño) }\end{array}$ \\
\hline
\end{tabular}

Es preciso mencionar que tan solo el $25 \%(\mathrm{n}=20)$ de los libros presentan alguna de estas ideas como un contenido explícito al describir qué caracteriza a la ciencia (figura 4). En el resto de casos aparecen asociadas con algún otro contenido, frecuentemente con las «etapas del método científico» (figura 5). En cualquier caso, sería aconsejable que todos los libros mostrasen la NdC, puesto que Manassero y Vázquez (1999) y Sardag et al. (2014) indican que debería formar parte del currículo de manera explícita.

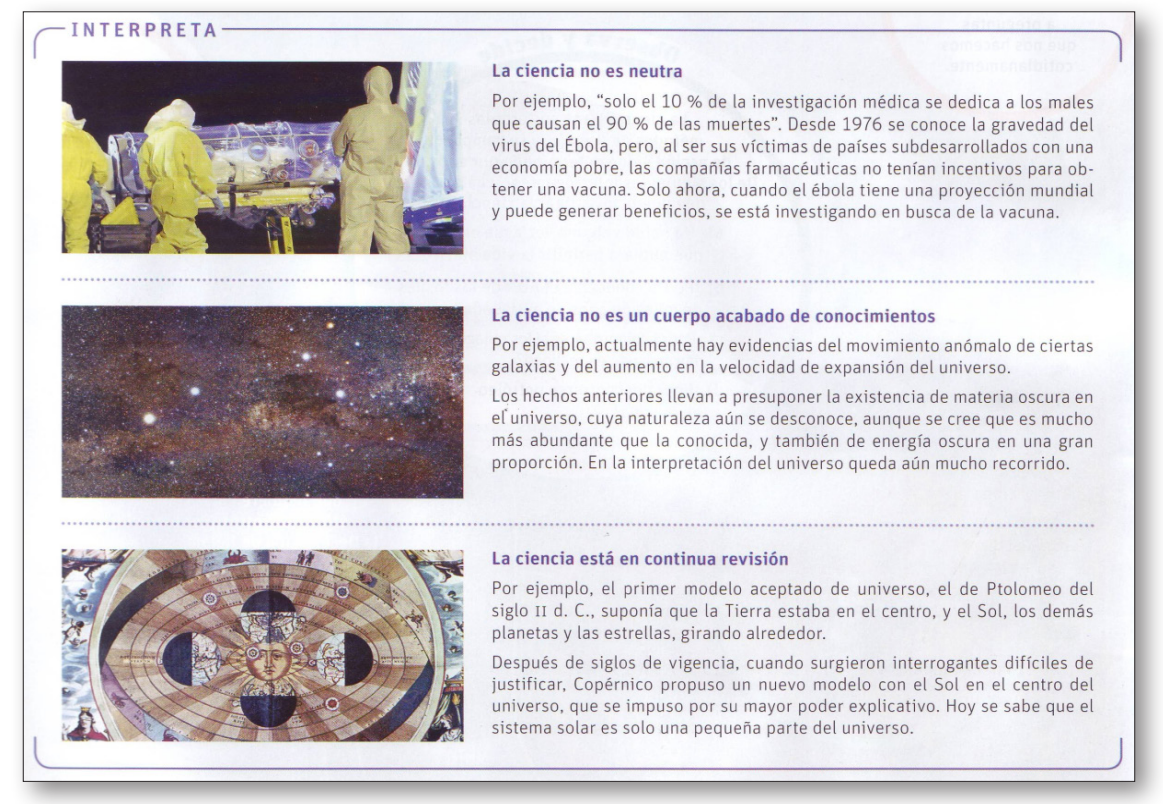

Fig. 4. Características de la ciencia. FQ, 3. ${ }^{\circ}$ ESO, 2015, SM (proyecto Savia) 


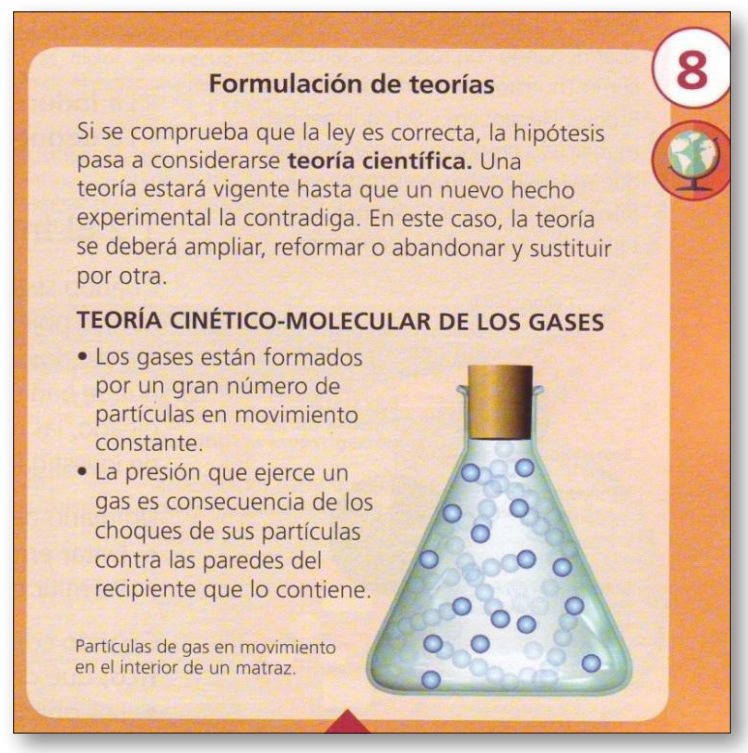

Fig. 5. Ejemplo de ciencia provisional y autocorregible. FQ, 3. ${ }^{\circ} \mathrm{ESO}, 2015$, Casals

Sobre la atención que se da a estos contenidos en las actividades, el $60 \%$ de los libros $(\mathrm{n}=20)$ no contiene ninguna actividad y en el resto hay como máximo tres. Esto nos hace pensar en la clara falta de reflexión sobre la ciencia, sobre la que Guisasola y Morentin (2007) ya han advertido.

\section{Enfrentamiento pseudociencia $v s$. ciencia}

En diez de los libros analizados se enfrenta la ciencia a la pseudociencia, pero solo ocho de ellos usan ese término o el de falsa ciencia. En el resto, en uno aparece como un ejercicio resuelto (figura 6) y en otro como la introducción de la portada al tema. En la tabla 4 se muestran las diferentes localizaciones.

Tabla 4

Localización del enfrentamiento pseudociencia $v$ s. ciencia $(\mathrm{n}=10)$

\begin{tabular}{|l|l|}
\hline \multicolumn{1}{|c|}{ Localización } & $\%$ \\
\hline En el cuerpo del texto sin encuadrar & 10 \\
\hline En el cuerpo del texto y con la definición encuadrada & 10 \\
\hline Tanto en el cuerpo del texto como en los márgenes y encuadrado & 10 \\
\hline En los márgenes y encuadrado & 50 \\
\hline Otras & 20 \\
\hline
\end{tabular}

Las definiciones de pseudociencia son muy escasas y suelen localizarse fuera del cuerpo principal del texto, pudiendo ser consideradas por el alumnado como poco importantes. Por lo general, la pseudociencia aparece descrita como aquello que se hace pasar por ciencia pero sin serlo, pues no cumple con las bases o métodos que caracterizarían a la ciencia: «sin base experimental o racional o científica» (BG, 1. ESO, 2015, Bruño) o «mal uso del método científico» (FQ, 3. ESO, 2016, Algaida). Enseñar qué es la pseudociencia debería ser relevante, porque solo un mayor conocimiento de la ciencia no implica un aumento del escepticismo frente la pseudociencia (Johnson y Pigliucci, 2004; Lundström y 
Jakobsson, 2009). Sin embargo, la cantidad de libros que advierte sobre la pseudociencia apenas roza la mitad, y se hace de forma somera. Aunque las descripciones encontradas son correctas, definiciones más extensas, con ejemplos concretos, cercanos y de repercusión social, como los relacionados con la salud, mejorarían su comprensión a la par que despertarían el análisis crítico del estudiante.

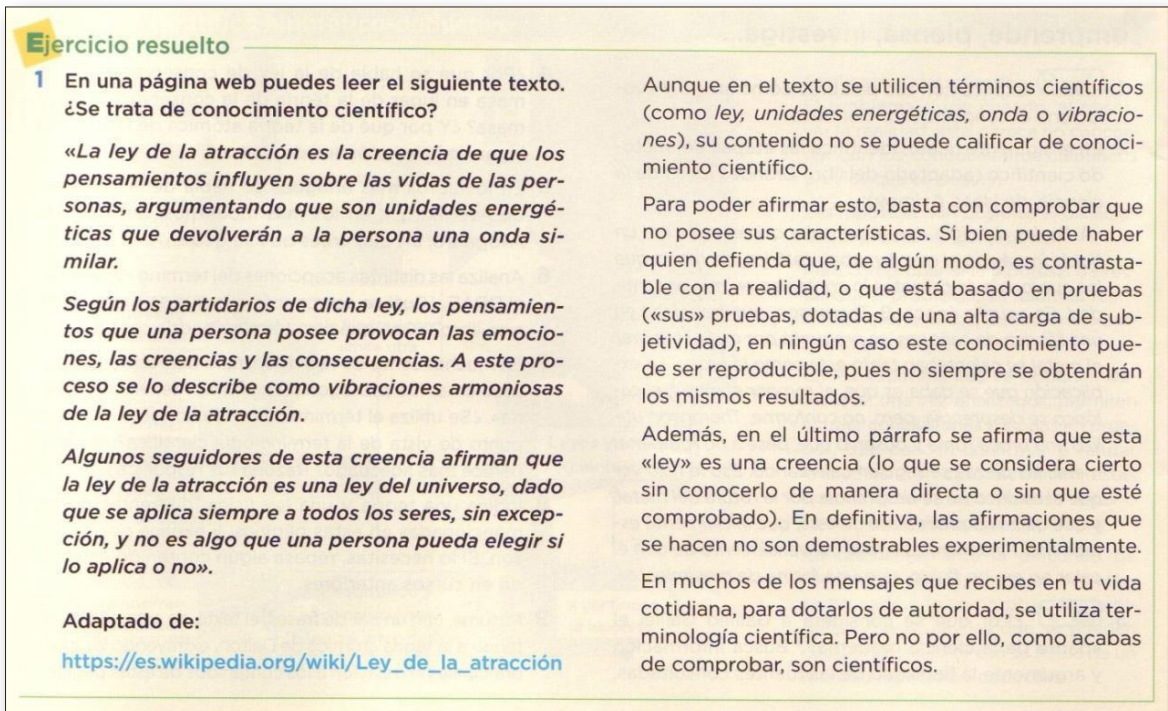

Fig. 6. Ejemplo de pseudociencia en ejercicio resuelto. FQ, 4. ${ }^{\circ}$ ESO, 2016, Anaya

A excepción de uno, en los libros en los que aparece este enfrentamiento hay al menos una actividad sobre ello. Este hecho puede compensar, en parte, la posible falta de atención sobre contenidos situados en los márgenes. No obstante, los ejercicios podrían ser algo más complejos, dirigiéndose, por ejemplo, a analizar e investigar un tópico pseudocientífico, como muestran Vílchez-González, Romero-López y González-García (2015). Sin embargo, muchos son una simple clasificación, científico vs. no científico -incluidos ejemplos de supersticiones, que no son pseudociencia-, o solo tratan el caso del horóscopo. Este hecho podría dar la falsa impresión de que discriminar la pseudociencia de la ciencia es algo sencillo. Pero lo cierto es que la pseudociencia puede alcanzar un alto nivel de sofisticación, lo que supone una gran dificultad para diferenciarla de la ciencia, incluso para los profesionales si esta se sale de su campo de estudio (Losh y Nzweske, 2011). Por lo tanto, conviene recalcar la importancia de contrastar siempre la información y recurrir a fuentes fiables. De esta manera, el tópico de la pseudociencia podría aprovecharse como un elemento original y llamativo para trabajar en el aula la búsqueda de información bibliográfica, clave en cualquier trabajo científico.

\section{Descripción de la metodología científica}

La metodología científica aparece en el $95 \%(\mathrm{n}=20)$ de los casos. Aunque un gran número de libros señala que en la ciencia no hay un único método de trabajo sino varios, existen diferentes errores o contradicciones (tabla 5):

- Algunos todavía muestran el método científico como único, ya que así lo llaman, y tampoco avisan ni muestran que no sea el único.

- «El método científico» es el título del tema en siete de los libros analizados, con independencia de que en ellos se avise, o no, de la existencia de diversidad metodológica. 
- Entre los libros que no lo presentan como el único existente, en varios aparece una visión contradictoria. Así, encontramos citas como la siguiente: «No existe un único método científico, pero sí hay una serie de etapas comunes que todos deberían seguir» (FQ, 3. ${ }^{\circ}$ ESO, 2015, McGrawHill Education).

- En todos los libros aparece desarrollado exclusivamente el método hipotético-deductivo.

Tabla 5.

Porcentaje de libros que cubren diversos

aspectos sobre metodología científica $(\mathrm{n}=19)(\%)$

\begin{tabular}{|l|l|}
\hline Único método & 16 \\
\hline Diversos métodos & 84 \\
\hline Hipotético-deductivo & 100 \\
\hline Esquema del método científico & 32 \\
\hline Descripción de etapas & 89 \\
\hline Ejemplos en etapas & 68 \\
\hline Presencia de actividades & 100 \\
\hline
\end{tabular}

Todo esto contribuye a una visión rígida del método científico como un conjunto de pasos que deben seguirse sí o sí. No obstante, hay tres libros que destacan positivamente por lo contrario. Así, en FQ, 3. ${ }^{\circ}$ ESO, 2015, Santillana, aparece lo siguiente: «Algún paso se puede repetir o no realizarse. Por ejemplo, los astrónomos solo miden lo que sucede en el universo, pero no pueden experimentar con él». También los libros de Bruño de BG de $1 .^{\circ}$ y $3 .^{\circ}$ avisan de que los experimentos no son siempre posibles. En cualquier caso, e incluso cuando sí se explica correctamente la existencia de una diversidad metodológica, echamos de menos ejemplos sobre esta, pues serían útiles para comprenderla mejor. Finalmente, cabe mencionar que dos libros hablan de dos métodos, inductivo y deductivo, pero incurren en errores al definirlos, denominando como inductivo lo que en realidad es hipotético-deductivo.

La descripción de las diferentes etapas de la metodología científica viene normalmente acompañada de ejemplos prácticos y conectados entre sí, los cuales, sin duda, ayudan a una mejor comprensión en comparación con aquellos que no los aportan. En el caso de los libros de Anaya de FQ de $2 .^{\circ}$ y $3 .^{\circ}$ de la ESO no se describen las etapas de la metodología científica, aunque sí se muestra un esquema de esta.

En torno a la metodología científica hemos encontrado con cierta frecuencia dos ideas poco correctas. La primera es otorgar a la experimentación un valor clave como fase del método científico, o como criterio de demarcación de la ciencia. Esta concepción es considerada por McComas (1998) como uno de los mitos de la ciencia. Y la segunda, no siempre cierta, y presente en aproximadamente la mitad de los libros, es la de que una hipótesis confirmada se convierte en una ley. Como afirma Woodcock (2014), no todas las cuestiones científicas aspiran a convertirse en principios generales, por lo tanto no todas las respuestas científicas tomarán forma de ley, sino que podrán hacerlo también como correlaciones, relaciones causales, modelos, etc.

La localización de la descripción de la metodología científica aparece siempre en el cuerpo del texto, con frecuencia hay definiciones que están encuadradas (79\%; $\mathrm{n}=19)$ y ocasionalmente (26\%; n = 19) se encuentra también algo en los márgenes. Respecto a las actividades analizadas, se observa que más de la mitad trabajan la metodología científica. 


\section{Presencia de modelos científicos}

Para analizar la presencia de modelos científicos, se diferenció entre la presencia de solo ejemplos concretos y cuando aparecían asociados a la necesidad que la ciencia tiene de ellos. Así, encontramos que el $35 \%$ de los libros $(n=20)$ hace referencia a la existencia de los modelos científicos como resultado del trabajo científico, limitándose cinco de ellos a dar la definición de modelo. Tan solo los libros de Anaya de $3 .^{\circ}$ y $4 .^{\circ}$ de la ESO de FQ van más allá, mostrando diferentes ejemplos ilustrados, explicando cómo pueden funcionar unos u otros modelos, su utilidad o no para según qué caso, su parte de «irreales»o los diferentes grados de complejidad posibles para representar una misma realidad (figura 7). En todos los casos esa definición aparece en el cuerpo del texto (encuadrada en tres casos), hecho que al menos resalta la importancia del concepto.

Comúnmente, los modelos vienen definidos como representaciones simplificadas de la realidad, si bien en dos libros se dice que es una «representación gráfica o mental que sirve para poder interpretar algo de lo que no se tiene certeza absoluta» (FQ, 3. ${ }^{\circ}$ y 4. ${ }^{\circ}$ ESO, 2015 y 2016, Edelvives). Esta última definición evitaría el mito de que los modelos científicos representan la realidad (McComas, 1998). En todo caso, una definición por sí sola no es suficiente para exponer la utilidad instrumental de los modelos científicos. Dicha utilidad solo se muestra adecuadamente en los ya mencionados libros de Anaya (figura 7).

En el $40 \%$ de los libros $(n=20)$ aparecen ejemplos ilustrados de algún modelo científico. De ellos, solo tres no llevan asociada la idea de que son el resultado del trabajo científico. El $25 \%$ (n = 20) contiene alguna actividad referente a estos, porcentaje menor al de los que hacen referencia teórica.

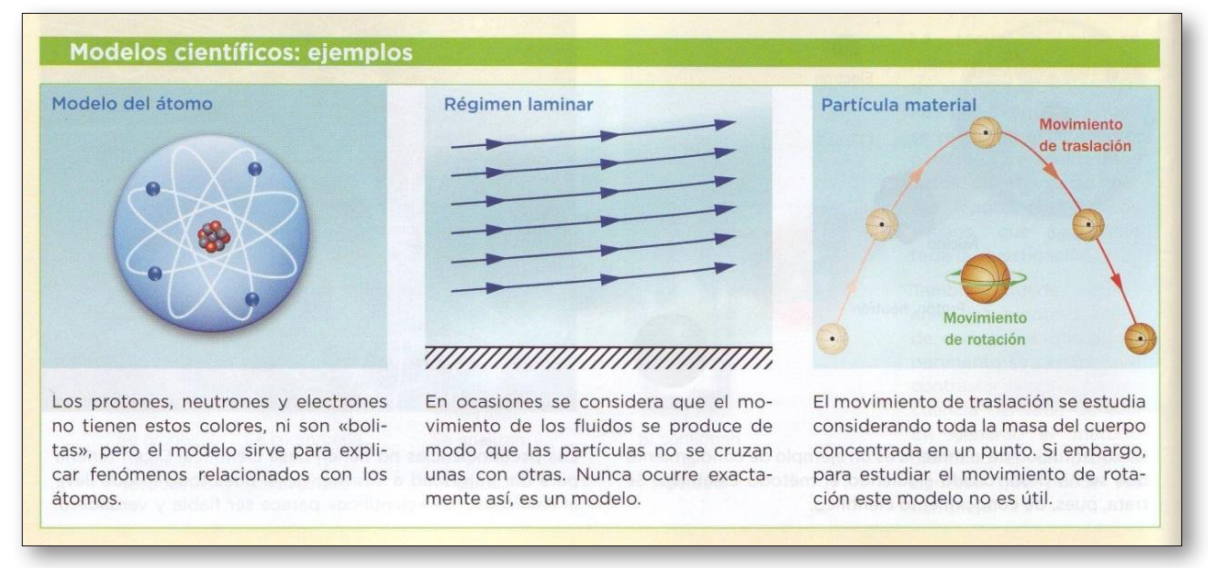

Fig. 7. Modelos científicos. FQ, 3. ESO, 2016, Anaya

\section{Evolución de la ciencia}

Se ha considerado que en los libros aparecía la evolución de la ciencia cuando, al menos, se mostraba con casos concretos como en el siguiente ejemplo: «el primer modelo aceptado de universo [...], suponía que la Tierra estaba en el centro [...] Después de siglos de vigencia [...] Copérnico propuso un nuevo modelo...» (FQ, 3. ${ }^{\circ}$ ESO, 2015, SM). El 55 \% (n = 20) muestra la evolución de la ciencia, pero limitándose casi todos ellos a dar un ejemplo. La mayoría ponen el del cambio del modelo geocéntrico al heliocéntrico o los sucesivos modelos de la teoría atómica. Dichos ejemplos aparecen en los libros como sigue: 
Gráfico 1.

Número de libros según localización

de la evolución de la ciencia $(\mathrm{n}=11)$

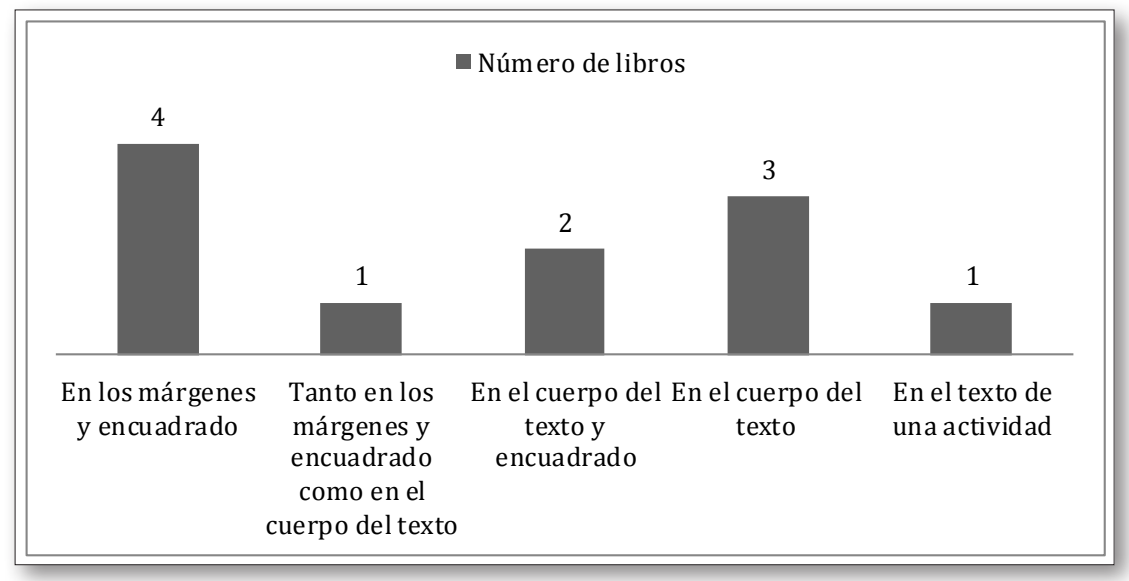

En relación con las actividades, se encuentran tan solo cuatro, en cuatro libros diferentes, una por cada uno. A la vista de los resultados, podemos decir que tanto la evolución de la ciencia como los modelos científicos reciben muy poca atención, y no solo por el bajo número de libros en los que aparecen, sino también por lo superfluo y anecdótico de su aparición. Algo parecido sucede con la alusión a la influencia del marco sociocultural e histórico (epígrafe 2). La historia y evolución de la ciencia podrían aprovecharse más, conectándolas tanto con la naturaleza de la ciencia como con la de la pseudociencia, ya que su utilidad en este sentido ha sido recomendada por varios autores (Allchin, 2012). Además, según Solbes y Traver (2001), su inclusión en la enseñanza de las ciencias aumenta la motivación y el interés de los alumnos. En este sentido, nos gustaría destacar el aporte que hacen, al reflejar la historia y evolución de la ciencia, los cómics Locos por la ciencia del libro de SM de BG de 1. ${ }^{\circ}$ de la ESO, dejando ver la influencia del marco cultural, así como la contribución de la mujer.

\section{Contribución de la mujer}

El $75 \%$ de los libros $(n=20)$ muestra, de alguna manera, la contribución de la mujer a la ciencia. Sin embargo, diez de ellos restringen exclusivamente a imágenes (en general de adorno) la aparición de la mujer, y solo en cinco de ellos se nombra a científicas concretas. Por otro lado, seis libros contienen hasta tres actividades (entre una y tres) que incluyen a la mujer. Por tanto, la contribución de la mujer a la ciencia en estos temas se ve poco reflejada. Este hecho fue anteriormente constatado por LópezNavajas (2014), quien analizó en libros de texto de todas las asignaturas de la ESO el porcentaje de personajes femeninos, lo que arrojó un resultado global del 12,8 \%. Para las asignaturas de Ciencias Naturales, BG y FQ, apenas se rozaba el $10 \%$. Aunque no podemos pretender que cuando se cuente la historia de la ciencia aparezcan tantas mujeres como hombres, sí podemos hacer reflexionar al alumnado sobre ello. Además, dicha reflexión puede aprovecharse para remarcar la influencia que el contexto sociocultural de cada momento tiene sobre cualquier actividad, incluida la científica, influencia que queda poco patente en los libros de texto (Campanile, Lederman y Kampourakis, 2015). 


\section{Análisis de actividades}

Para las actividades se analizaron dos aspectos: contenido trabajado y tipo de actividad, modificándose y ampliándose la clasificación de Koch y Eckstein (1991, citado en Campanario, 2001).

Tabla 6.

Categorías para la clasificación de actividades

\begin{tabular}{|c|c|}
\hline Tipos & Ejemplos extraídos de los libros analizados \\
\hline $\begin{array}{l}\text { Reproductivas (Reprod.): preguntas cuya respuesta presenta } \\
\text { una única solución que puede obtenerse a partir del texto. }\end{array}$ & $\begin{array}{l}\text { ¿En qué consiste un experimento controlado? (BG, } 1 .^{\circ} \text { de la } \\
\text { ESO, 2015, Bruño). }\end{array}$ \\
\hline $\begin{array}{l}\text { Deductivas (Deduct.): preguntas cuya respuesta presenta } \\
\text { una única solución que puede deducirse a partir del texto. } \\
\text { Tienen un carácter más teórico que las aplicadas. }\end{array}$ & $\begin{array}{l}\text { ¿Qué diferencias hay entre una ley y una hipótesis? ¿Y entre } \\
\text { una ley y una teoría científica? (Física y Química, 3. }{ }^{\circ} \text { de la } \\
\text { ESO, 2016, Oxford). }\end{array}$ \\
\hline $\begin{array}{l}\text { Aplicadas (Aplicad.): preguntas cuya respuesta presenta } \\
\text { una solución que conlleva la aplicación del conocimiento } \\
\text { presentado en el texto (ejemplos: problemas numéricos; } \\
\text { interpretación de gráficos; o actividades creativas donde se } \\
\text { pida, por ejemplo, generar un modelo explicativo o diseñar } \\
\text { un experimento). Si bien la respuesta no es necesariamente } \\
\text { única, sí habrá soluciones correctas e incorrectas. Tienen un } \\
\text { carácter más práctico que las deductivas. }\end{array}$ & $\begin{array}{l}\text { Diseńa un experimento para comprobar la veracidad de la } \\
\text { siguiente hipótesis: «Los hongos, como el moho del pan o de } \\
\text { la fruta, crecen mejor cuando la temperatura y la humedad } \\
\text { ambiental son elevadas» (BG, } 1 .^{\circ} \text { de la ESO, 2016, Oxford). } \\
\text { ¿A qué temperatura se disuelven } 275 \text { g de azúcar en } 100 \mathrm{~g} \text { de } \\
\text { agua? (FQ, 3. } .^{\circ} \text { de la ESO, 2016, Oxford). }\end{array}$ \\
\hline $\begin{array}{l}\text { Prácticas o experimentales (Pr.-Exp.): se realiza alguna prác- } \\
\text { tica o experimento. Con frecuencia implican aplicar uno o } \\
\text { varios pasos de la metodología científica. }\end{array}$ & $\begin{array}{l}\text { Siguiendo los pasos del método científico, comprueba si la } \\
\text { siguiente hipótesis es cierta: "Cuanta más agua tiene una } \\
\text { planta, más crece» (BG, 1. }{ }^{\circ} \text { de la ESO, 2016, Oxford). }\end{array}$ \\
\hline $\begin{array}{l}\text { Lógico-reflexivas (L-R): se plantea una pregunta que no } \\
\text { tiene una única solución y que precisa razonar o pensar en } \\
\text { las posibles respuestas. La respuesta exigida (pedida) tiene } \\
\text { un carácter más subjetivo o personal que en las crítico-ar- } \\
\text { gumentativas. }\end{array}$ & $\begin{array}{l}\text { ¿Qué diferencias crees que existen entre una observación y } \\
\text { un experimento? (FQ, } 3 .^{\circ} \text { de la ESO, 2016, Oxford). }\end{array}$ \\
\hline $\begin{array}{l}\text { Crítico-argumentativas (Crit.-Arg.): se trabaja sobre una si- } \\
\text { tuación o afirmación ya dada, para indagar en su veracidad, } \\
\text { pidiéndose un análisis u opinión justificada con argumen- } \\
\text { tos. La respuesta exigida tiene un carácter más objetivo que } \\
\text { en las lógico-reflexivas (pues debe basarse en algo más que en } \\
\text { una mera creencia u opinión). }\end{array}$ & $\begin{array}{l}\text { Comenta la veracidad de la siguiente afirmación: «En caso } \\
\text { de que el tiempo sea una de las variables de nuestra experi- } \\
\text { mentación, siempre irá en el eje horizontal de las gráficas» } \\
\text { (BG, } 1 .^{\circ} \text { de la ESO, 2016, Oxford). }\end{array}$ \\
\hline $\begin{array}{l}\text { De búsqueda bibliográfica (B. Biblio.): se necesita informa- } \\
\text { ción que no está en el texto, o se pide explícitamente la bús- } \\
\text { queda de información extra, que no viene en el texto. Una } \\
\text { vez encontrada la información requerida, se podrá encuadrar } \\
\text { simultáneamente en alguno de los otros tipos de actividades. }\end{array}$ & $\begin{array}{l}\text { Busca en libros o en internet los nombres de tres científicos } \\
\text { o científicas importantes y señala alguno de sus descubri- } \\
\text { mientos (FQ, 3. }{ }^{\circ} \text { de la ESO, 2015, Bruño). }\end{array}$ \\
\hline $\begin{array}{l}\text { Otras: aquellas que no se pueden clasificar en ninguno de } \\
\text { los tipos anteriores. }\end{array}$ & $\begin{array}{l}\text { Más del } 50 \% \text { de la población mundial vive en las ciudades } \\
\text { y los documentales nos acercan a la naturaleza. Conocerla } \\
\text { nos permite protegerla. Busca en YouTube algunos vídeos de } \\
\text { Félix Rodríguez de la Fuente y otros más actuales, y disfruta } \\
\text { con ellos (BG, } 1 .^{\circ} \text { de la ESO, 2015, SM). }\end{array}$ \\
\hline
\end{tabular}

Puesto que una actividad puede estar en más de una categoría simultáneamente, la suma de los porcentajes puede superar el $100 \%$. Por su peculiaridad, hemos considerado aparte aquellas tareas que involucran al estudiante en una investigación o práctica, que suelen aparecer al final y que hemos denominado sección final. Cada sección se ha contabilizado como una sola actividad. En ninguna de 
estas secciones hay relación desde el punto de vista teórico con los contenidos analizados, por lo que no se presenta para ellas clasificación por contenidos.

En aquellos casos clasificados como "Parte de un tema» solo se analizaron las actividades relativas a esa parte del tema, mientras que en aquellos clasificados como «Tema completo» se analizaron todas las actividades del tema.

\section{Contenido trabajado}

En la mayoría de los casos las actividades analizadas no trabajan más de un contenido simultáneamente. Tan solo se hallaron cuatro que, al trabajar la metodología científica, lo hacían junto a alguna característica de la $\mathrm{NdC}$ y otra actividad que trabaja la evolución de la ciencia mediante los modelos. El número de actividades que trabaja cada concepto está en consonancia con lo encontrado a nivel teórico. Hay un alto número de actividades que trabaja la metodología científica, mientras que los conceptos de ciencia, modelo, evolución de la ciencia y contribución de la mujer reciben menor atención (tabla 7). El porcentaje de actividades sobre pseudociencia, si bien no es alto, ha sido algo mayor de lo que esperábamos, teniendo en cuenta los contenidos teóricos encontrados.

Tabla 7.

Porcentaje de actividades que trabaja cada contenido $(\mathrm{n}=455)(\%)$

\begin{tabular}{|c|c|c|c|c|c|c|}
\hline Ciencia & Modelo & MC & Ps vs. C & Evolución & Mujer & Otro \\
\hline 3,7 & 2,2 & 59,1 & 4,8 & 0,9 & 2,2 & 27,9 \\
\hline
\end{tabular}

Notas: $\mathrm{MC}=$ método científico. Ps vs. $\mathrm{C}=$ enfrentamiento entre pseudociencia y ciencia.

\section{Tipo de actividad}

El 18,9 \% de las actividades se ha clasificado simultáneamente en más de un tipo. El número de actividades crítico-argumentativas es bajo en todos los libros (tabla 8), aun cuando el uso de la argumentación ha demostrado beneficios en la enseñanza en general (Yu y Yore, 2013) y como recurso para disminuir las creencias pseudocientíficas en particular (Tsai et al., 2015). Lo mismo sucede con las práctico-experimentales. Si bien en este último caso podría compensarse un poco en aquellos libros que tengan secciones finales con actividades de este tipo (tabla 9). En los libros de Bruño de BG de 1. ${ }^{\circ}$ y $3 .^{\circ}$ de la ESO y de FQ de $2 .^{\circ}$ son principalmente reproductivas, suponiendo la mitad de las actividades en el libro de $2 .^{\circ}$ y más de la mitad en los de $1 .^{\circ}$ y $3 .^{\circ}$.

Tabla 8 .

Porcentaje de actividades que se encuadra dentro de cada tipo $(\mathrm{n}=455)(\%)$

\begin{tabular}{|c|c|c|c|c|c|c|c|}
\hline Reprod. & Deduct. & Aplicad. & Pr.-Exp. & L-R & Crit.-Arg. & B. Biblio. & Otras \\
\hline 27,9 & 23,5 & 22,0 & 3,5 & 20,4 & 2,2 & 17,4 & 5,0 \\
\hline
\end{tabular}

En las secciones finales predominan las actividades aplicadas y práctico-experimentales (tabla 9), hecho que era de esperar por la naturaleza de estas secciones (protocolos o guías prácticas y/o de investigación). En 7 de estas 22 secciones analizadas se les pide que elaboren un informe sobre lo realizado. Dicha petición permite asentar los conocimientos y acercar al estudiante a la difusión del conocimiento, procedimiento habitual dentro de la metodología científica. Finalmente, cabe señalar que estas 22 secciones se encuentran repartidas entre 13 libros (12 de FQ y 1 de BG) y que el porcentaje de secciones que trabaja más de un tipo de actividad es del $91 \%$. 
Tabla 9.

Tipo de actividades trabajadas en las secciones finales $(n=22)(\%)$

\begin{tabular}{|c|c|c|c|c|c|c|c|}
\hline Reprod. & Deduct. & Aplicad. & Pr.-Exp. & L-R & Crit.-Arg. & B. Biblio. & Otras \\
\hline 4 & 18 & 86 & 68 & 32 & 0 & 27 & 14 \\
\hline
\end{tabular}

\section{CONCLUSIONES}

Los libros de texto suelen ser un reflejo del currículo oficial y de uso recurrente en las aulas. Esto hace que sean objeto imprescindible de análisis en la investigación de la Didáctica de las Ciencias, puesto que forman parte esencial de su enseñanza y aprendizaje. Así se posibilita la reflexión y discusión para su mejora y especialmente para ayudar a tomar decisiones al profesorado.

En el currículo de ciencias de la ESO se incluye un tema de introducción a la ciencia en todos los cursos, salvo para BG de $4 .^{\circ}$. Este hecho marca los contenidos que presentan las diversas editoriales. Tratándose de un tema esencial para introducir al alumnado en las ciencias, ver cómo se trabaja en ellas y su relación con su vida diaria, debe ser un objetivo de interés conocer qué visión de ciencia se muestra en dicho tema. Y este ha sido el que hemos pretendido cubrir con la presente investigación.

De lo dicho anteriormente cabría esperar que se ofreciera una imagen adecuada sobre la NdC. Sin embargo, la visión que se presenta es manifiestamente mejorable. Así, varios libros, principalmente los de BG, excluyen el tema de introducción a la ciencia, y cuando aparece, presenta ciertas deficiencias en los contenidos investigados, excepto para el método científico, que es el principal protagonista. Sin embargo, con cierta frecuencia viene acompañado de algunas concepciones erróneas o sesgadas sobre la metodología científica que pueden afianzar ideas equivocadas entre el alumnado. Suelen aparecer con frecuencia algunas características de la $\mathrm{NdC}$, pero no se enfrentan al concepto de pseudociencia, dejándose de lado la reflexión sobre qué es la ciencia y cómo funciona, algo esencial para comprenderla. Respecto a las actividades, se echa en falta más presencia de las que requieren argumentación y pensamiento crítico, ambos necesarios para formar a ciudadanos responsables.

No obstante lo dicho, reconocemos como una limitación de este análisis el que esté circunscrito al tema de la introducción a la ciencia. Desconocemos si los resultados con respecto a la atención que reciben algunos de los conceptos analizados serían mejores en caso de que se hubiesen estudiado en todo el libro. Se presenta así una posible línea de investigación futura.

Si bien los libros son un vehículo para el proceso de enseñanza-aprendizaje, los guías de ese proceso son los profesores. Por este motivo, investigaciones como la que aquí se presenta deben tenerse en cuenta especialmente en la formación inicial del profesorado de Secundaria. Como sugerencia, se propone utilizar estos temas introductorios de libros de texto de ciencias para que los alumnos del Máster de Profesorado realicen un análisis crítico y reflexivo y establezcan comparaciones entre ellos.

No queremos finalizar sin incluir una reflexión personal que creemos pertinente. Si el currículo oficial es el que guía los contenidos que aparecen en los libros de texto, llama la atención que aparezcan diferencias entre el de FQ y el de BG, lo que podría contribuir a propiciar una imagen inadecuada de la $\mathrm{NdC}$. Al consultarlo nos surgen varias preguntas: ¿por qué en el $4 .^{\circ}$ curso no hay un tema introductorio para BG y sí lo hay para FQ?, ¿por qué siendo un tema introductorio en ambas asignaturas no existe una parte común en el enunciado de ambas?, ¿por qué para FQ se habla del método científico y de sus pasos, mientras que en BG se habla de metodología científica?, ¿es que no se miden magnitudes en la biología ni en la geología?, ¿los experimentos solo se realizan en los laboratorios? Sería deseable, por lo tanto, que los resultados de estas investigaciones trasciendan también a quienes se encargan de legislar, dado que ello será la pauta para posibles cambios en los contenidos que presenten las editoriales. 


\section{AGRADECIMIENTOS}

Agradecemos a Glenn Harding su amable revisión del inglés, y a los dos revisores anónimos sus comentarios para mejorar este artículo.

\section{REFERENCIAS BIBLIOGRÁFICAS}

Acevedo-Díaz, J. A. (2009). Enfoques explícitos versus implícitos en la enseñanza de la naturaleza de la ciencia. Revista Eureka sobre Enseñanza y Divulgación de las Ciencias, 6(3), 355-386.

https://doi.org/10.25267/rev_eureka_ensen_divulg_cienc.2009.v6.i3.04

Acevedo-Díaz, J. A. y García-Carmona, A. (2016). "Algo antiguo, algo nuevo, algo prestado». Tendencias sobre la naturaleza de la ciencia en la educación científica. Revista Eureka sobre Enseñanza y Divulgación de las Ciencias, 13(1), 3-19.

https://doi.org/10.25267/rev_eureka_ensen_divulg_cienc.2016.v13.i1.02

Acevedo-Díaz, J. A., García-Carmona, A. y Aragón, M. M. (2017). Historia de la ciencia para enseñar naturaleza de la ciencia: una estrategia para la formación inicial del profesorado de ciencia. Educación Química, 28(3), 140-146. https://doi.org/10.1016/j.eq.2016.12.003

Afonso, A. S. y Gilbert, J. K. (2010). Pseudo-science: A meaningful context for assessing nature of science. International Journal of Science Education, 32(3), 329-348. https://doi.org/10.1080/09500690903055758

Allchin, D. (2012). Teaching the nature of science through scientific errors. Science Education, 96(5), 94-926. https://doi.org/10.1002/sce.21019

Alonso-Marcos, F. y Cortiñas-Rovira, S. (2014). La pseudociencia y el poder de los medios de comunicación. La problemática ausencia de bases teóricas para afrontar el fenómeno. Historia y Comunicación Social, 19, 93-103.

https://doi.org/10.5209/rev_hics.2014.v19.45111

Bermúdez, G. (2015). Los orígenes de la Biología como ciencia. El impacto de las teorías de evolución y las problemáticas asociadas a su enseñanza y aprendizaje. Revista Eureka sobre Enseñanza y Divulgación de las Ciencias, 12(1), 66-90.

https://doi.org/10.25267/rev_eureka_ensen_divulg_cienc.2015.v12.i1.06

Bian, L., Leslie, S. J. y Cimpian, A. (2017). Gender stereotypes about intellectual ability emerge early and influence children's interests. Science, 355(6323), 389-391.

https://doi.org/10.1126/science.aah6524

Campanario, J. M. (2001). ¿Qué puede hacer un profesor como tú o un alumno como el tuyo con un libro de texto como éste? Una relación de actividades poco convencionales. Enseñanza de las Ciencias, 19(3), 351-364.

Campanile, M. F., Lederman, N. G. y Kampourakis, K. (2015). Mendelian Genetics as a Platform for Teaching About Nature of Science and Scientific Inquiry: The Value of Textbooks. Science \& Education, 24, 205-225. https://doi.org/10.1007/s11191-013-9607-4

Centinkaya, E., Turgut, H. y Duru, M. K. (2015). The Effect of the Context of Science, Pseudoscience Demarcation on the Science Perceptions of Secondary School Students: The Case of Iridology. Egitim ve Bilim, 40(181), 1-18.

https://doi.org/10.15390/eb.2015.3127 
Clough, M. P. (2006). Learners' Responses to the Demands of Conceptual Change: Considerations for Effective Nature of Science Instruction. Science \& Education, 15(5), 463-494. https://doi.org/10.1007/s11191-005-4846-7

Domènech-Casal, J. (2015). Una secuencia didáctica de modelización, indagación y creación del conocimiento científico en torno a la deriva continental y la tectónica de placas. Revista Eureka sobre Enseñanza y Divulgación de las Ciencias, 12(1), 186-197. https://doi.org/10.25267/rev_eureka_ensen_divulg_cienc.2015.v12.i1.13

Galagovski, L. y AdúRiz-Bravo, A. (2001). Modelos y analogías en la enseñanza de las Ciencias Naturales. El concepto de modelo didáctico analógico. Enseñanza de las Ciencias, 19(2), 231-242.

GonzÁlez-Galli, L. (2010). Qué ciencia enseñar. Educar en ciencias (pp. 59-94). Buenos Aires, Paidós.

Good, R. (2012). Why the study of pseudoscience should be included in nature of science studies. En M. S. Khine (Ed.), Advances in Nature of Science Research (pp. 97-106). Springer Netherlands. https://doi.org/10.1007/978-94-007-2457-0_5

Guisasola, J. y Morentin, M. (2007). Comprenden la naturaleza de la ciencia los futuros maestros y maestras de Educación Primaria? Revista Electrónica de Enseñanza de las Ciencias, 6(2), 246-262.

Johnson, M. y Pigliucci, M. (2004). Is knowledge of science associated with higher skepticism of pseudoscientific claims? The American Biology Teacher, 66(8), 536-548. https://doi.org/10.2307/4451737

Khishfe, R. y AbD-El-Khalick, F. (2002). Influence of Explicit and Reflective versus Implicit Inquiry-Oriented Instruction on Sixth Graders' Views of Nature of Science. Journal of Research in Science Teaching, 39(7), 551-578. https://doi.org/10.1002/tea.10036

Koskal, M. S., Cakiroglu, J. y Geban, O. (2013). The effect of explicit embedded reflective instruction on nature of science understangdings in advanced students. Journal of Biological Education, $47(4), 208-223$. https://doi.org/10.1080/00219266.2013.799080

Lederman, N. G. (2007). Nature of Science: Past, Present and Future. En S. K. Abell y N. G. Lederman (Eds.), Handbook of research on science education (pp. 831-879). Mahwah, NJ: Lawrence Erlbaum Associates.

Lederman, N. G. y Lederman, J. S. (2014). Is Nature of Science Going, Going, Going, Gone? Journal of Science Teacher Education, 25, 235-238. https://doi.org/10.1007/s10972-014-9386-z

López-Navajas, A. (2014). Análisis de la ausencia de las mujeres en los manuales de la ESO: una genealogía de conocimiento ocultada. Revista de Educación, 363, 282-308. https://doi.org/10.4438/1988-592X-RE-2012-363-188

Losh, S. C. y Nzekwe, B. (2011). Creatures in the Classroom: Preservice Teacher Beliefs About Fantastic Beasts, Magic, Extraterrestrials, Evolution and Creationism. Science \& Education, 20(5-6), 473-489. https://doi.org/10.1007/s11191-010-9268-5

Lundström, M. у Jakobsson, A. (2009). Students' ideas regarding science and pseudo-science in relation to the human body and health. Nordic Studies in Science Education, 5(1), 3-17. https://doi.org/10.5617/nordina.279

Manassero-Mas, M. A. y Vázquez-Alonso, Á. (1999). Ideas de los estudiantes sobre la epistemología de la ciencia: modelos, leyes y teorías. Revista de Educación, 320, 309-334. 
McComas, W. F. (1998). The principal elements of the nature of science: Dispelling the myths. En W. F. McComas (Ed.), The Nature of Science in Science Education (pp. 53-70). The Netherlands: Kluwer Academic Publishers. https://doi.org/10.1007/0-306-47215-5_3

McComas, W. F. (2003). A Textbook Case of the Nature of Science: Laws and Theories in the Science of Biology. International Journal of Science and Mathematics Education, 1(2), 141-155. https://doi.org/10.1023/b:ijma.0000016848.93930.9c

McComas, W. F., Clough, M. P. y Almazroa, H. (1998). The role and character of the nature of science in science education. En W. F. McComas (Ed.), The Nature of Science in Science Education (pp. 3-39). Holanda: Kluwer Academic Publishers. https://doi.org/10.1007/0-306-47215-5_1

Occelli, M. y Valeiras, N. (2013). Los libros de texto de ciencias como objeto de investigación: una revisión bibliográfica. Enseñanza de las Ciencias, 31(2), 133-152.

Oliva, J. M., Aragón-Méndez, M. M., Jiménez-Tenorio, N. y Aragón-Núñez, L. (2016). La modelización en ciencias como estrategia de investigación y de intervención docente. Obtenido de: https://miriadax.net/documents/71899562/0/Modelizacion+en+Ciencias.pdf/80fa14d2-38964f88-bb9a-03efa9a62587

Orden de 14-7-2016, por la que se desarrolla el currículo correspondiente a la Educación Secundaria Obligatoria en la Comunidad Autónoma de Andalucía, se regulan determinados aspectos de la atención a la diversidad y se establece la ordenación de la evaluación del proceso de aprendizaje del alumnado (BOJA n.o 144, de 28/07/2016).

Park, H., Nielsen, W. y Woodruff, E. (2014). Students' Conceptions of the Nature of Science: Perspectives from Canadian and Korean Middle School Students. Science \& Education, 23(5), 1169-1196. https://doi.org/10.1007/s11191-013-9613-6

Real Decreto 1105/2014, de 26 de diciembre, por el que se establece el currículo básico de la Educación Secundaria Obligatoria y del Bachillerato (BOE 03/01/2015) (MECD, 2014).

Sardag, M., Aydin, S., Kalender, N., Tortumlu, S., Çiftçi, M. y Perihanoglu, S. (2014). The Integration of Nature of Science in the New Secondary Physics, Chemistry and Biology Curricula. Education and Science, 39(174), 233-248.

https://doi.org/10.15390/eb.2014.3069

Sjøberg, S. y Schreiner, C. (2010). The ROSE project. An overview and key findings. Obtenido de: https://www.roseproject.no/network/countries/norway/eng/nor-Sjoberg-Schreiner-overview-2010. pdf

Solaz-Portolés, J. J. (2010). La naturaleza de la ciencia y los libros de texto de ciencias: una revisión. Educación XX1, 13(1), 65-80. https://doi.org/10.5944/educxx1.13.1.277

Solbes, J. y Traver, M. (2001). Resultados obtenidos introduciendo historia de la ciencia en las clases de física y química: mejora de la imagen de la ciencia y desarrollo de actitudes positivas. Enseñanza de las Ciencias, 19(1), 151-162.

Tsai, C. Y., Lin, C. N., Shin, W. L. y Wu, P. L. (2015). The effect of online argumentation upon students'pseudoscientific beliefs. Computers \& Education, 80, 187-197. https://doi.org/10.1016/j.compedu.2014.08.018

VÁzquez-Alonso, Á. y Manassero-Mas, M. A. (2009). La relevancia de la educación científica: actitudes y valores de los estudiantes relacionados con la ciencia y la tecnología. Enseñanza de las Ciencias, 27(1), 33-48. 
VÁzquez-Alonso, Á. y Manassero-Mas, M. A. (2012). La selección de contenidos para enseñar naturaleza de la ciencia y tecnología (parte 1): Una revisión de las aportaciones de la investigación didáctica. Revista Eureka sobre Enseñanza y Divulgación de las Ciencias, 9(1), 2-31. https://doi.org/10.25267/rev_eureka_ensen_divulg_cienc.2012.v9.i1.02

Vílchez-González, J. M., Carrillo-Rosúa, J., Rodríguez-Sabiote, C. y Jiménez-Tejada, M. P. (2015). Imagen de ciencia de estudiantes de Magisterio. Didáctica de las ciencias experimentales y sociales, 29, 157-172.

https://doi.org/10.7203/dces.29.4283

Vílchez-González, J. M., Romero-López, M. C. y GonzÁlez-García, F. (2015). Científicamente demostrado... por el momento. Alambique: Didáctica de las ciencias experimentales, 81, 17-24.

Woodcock, B. A. (2014). "The Scientific Method» as Myth and Ideal. Science \& Education, 23(10), 2069-2093.

https://doi.org/10.1007/s11191-014-9704-z

Yu, S. M. y Yore, L. D. (2013). Quality, evolution, and positional change of university students' argumentation patterns about organic agriculture during an argument-critique-argument experience. International Journal of Science and Mathematics Education, 11(5), 1233-1254.

https://doi.org/10.1007/s10763-012-9373-9 


\section{ANEXO I}

Lista de libros analizados. Resaltando en gris aquellos en los que se han encontrado contenidos del tema de introducción a la ciencia.

\begin{tabular}{|c|c|c|c|}
\hline Asignatura & Editorial & Curso & Año \\
\hline \multirow{15}{*}{ Biología y Geología } & \multirow{2}{*}{ Algaida } & $1 .^{\circ}$ & \multirow{2}{*}{2016} \\
\hline & & $3 .^{\circ}$ & \\
\hline & \multirow{3}{*}{ Anaya } & $1 .^{o}$ & \multirow{3}{*}{2016} \\
\hline & & $3 .^{\circ}$ & \\
\hline & & $4.0^{\circ}$ & \\
\hline & \multirow{2}{*}{ Bruño } & $1 .^{\circ}$ & \multirow{2}{*}{2015} \\
\hline & & $3 .^{\circ}$ & \\
\hline & \multirow{2}{*}{ Casals } & $1 .{ }^{\circ}$ & 2016 \\
\hline & & $3 .^{\circ}$ & 2015 \\
\hline & Edebé & $1 .^{\circ}$ & 2015 \\
\hline & Edelvives & $1 .^{\circ}$ & 2016 \\
\hline & Oxford & $1 .{ }^{\circ}$ & 2016 \\
\hline & Santillana & $3 .{ }^{\circ}$ & 2015 \\
\hline & SM (proyecto Savia). Serie Arce & $1 .^{\circ}$ & 2015 \\
\hline & SM (proyecto Savia). Serie Arce & $3 .^{\circ}$ & 2015 \\
\hline \multirow{16}{*}{ Física y Química } & Algaida & $3 . .^{\circ}$ & 2016 \\
\hline & \multirow{3}{*}{ Anaya } & $2 .^{\circ}$ & \multirow{3}{*}{2016} \\
\hline & & $3 .^{\circ}$ & \\
\hline & & $4 .^{\circ}$ & \\
\hline & \multirow{2}{*}{ Bruño } & $2 .{ }^{\circ}$ & 2016 \\
\hline & & $3 .^{\circ}$ & 2015 \\
\hline & Casals & $3 .^{\circ}$ & 2015 \\
\hline & Edebé & $3 .^{\circ}$ & 2015 \\
\hline & \multirow{2}{*}{ Edelvives } & $3 .^{\circ}$ & 2015 \\
\hline & & $4 .^{\circ}$ & 2016 \\
\hline & McGrawHill Education & $3 .^{\circ}$ & 2015 \\
\hline & Oxford & $3 .^{\circ}$ & 2016 \\
\hline & Santillana & $2 .^{\circ}$ & 2016 \\
\hline & Santillana & $3 .^{\circ}$ & 2015 \\
\hline & SM (proyecto Savia) & $3 .^{\circ}$ & 2015 \\
\hline & Vicens Vives & $3 .^{\circ}$ & 2016 \\
\hline
\end{tabular}




\section{ANEXO II}

\section{Protocolo de análisis}

\begin{tabular}{|c|}
\hline Datos del libro \\
\hline $\begin{array}{l}\text { Asignatura: } \\
\text { Editorial: } \\
\text { Curso: } \\
\text { Título: } \\
\text { Año de edición: }\end{array}$ \\
\hline 1. Existe un bloque de contenidos dedicado a la introducción a la ciencia: SÍ \\
\hline $\begin{array}{l}\text { > Tipo de sección: } \\
\begin{array}{llll}\text { a) Apéndice } & \text { b) Parte de un tema } & \text { c) Tema completo } & \text { d) Otros }\end{array} \\
>\text { Número páginas/páginas totales libro: } \\
>\text { N. }{ }^{\circ} \text { tema/total: }\end{array}$ \\
\hline 2. Definición de ciencia: SÍ NO Descripción: SÍ NO \\
\hline $\begin{array}{l}\text { Marcar si en el libro aparecen ideas que contradigan alguna de las siguientes características de la NdC: } \\
\text { - La ciencia está basada en evidencia empírica. } \\
\text { - El método científico no es rígido y exclusivo. Existen diferentes métodos de investigación. } \\
\text { - La ciencia tiene un carácter tentativo. El conocimiento científico es provisional y autocorregible. } \\
\text { - El conocimiento científico es parcialmente subjetivo. Además el conocimiento científico es también producto de la } \\
\quad \text { imaginación y la creatividad humana. } \\
\text { - El conocimiento científico se ve afectado por el marco sociocultural e histórico. } \\
\text { - La ciencia no puede contestar a todo, existen límites. }\end{array}$ \\
\hline $\begin{array}{l}\text { > Localización del concepto de ciencia: • pie de imagen } \bullet \text { texto } \\
\text { > Localización dentro del texto: } \\
\begin{array}{ll}\text { a) En el cuerpo del texto y encuadrado } & \text { b) En el cuerpo del texto } \\
\text { c) En los márgenes y encuadrado } & \text { d) En los márgenes }\end{array} \\
\text { - Cantidad de actividades (n.o/total): } \\
\text { - Tipo: }\end{array}$ \\
\hline 3. Enfrentamiento pseudociencia vs ciencia: SÍ $\quad N O$ \\
\hline $\begin{array}{l}>\text { Ejemplo de pseudociencia: Sí } \quad \text { NO } \\
\left.>\text { Los ejemplos que aparecen están relacionados con: a) Salud (e indicar el n. }{ }^{\circ} \text { ) b) Otros (n. }{ }^{o}\right) \\
>\text { Localización: } \bullet \text { pie de imagen } \bullet \text { texto } \\
>\text { Localización dentro del texto: (ver ítem } 2) \\
\text { - Cantidad de actividades (n. }{ }^{\circ} / \text { total): } \\
\text { - Tipo: }\end{array}$ \\
\hline 4. Descripción de la metodología cientifica: SÍ NO \\
\hline $\begin{array}{l}\bullet \text { Único método • Diversos métodos } \\
\text { Tipos: • Inductivo } \bullet \text { Hipotético-deductivo } \bullet \text { Otros } \\
\text { > Localización: • esquema } \bullet \text { texto } \\
\text { > Ejemplificación práctica de las etapas: SÍ NO } \\
\text { > Localización dentro del texto: } \\
\text { - Cantidad de actividades }\left(\mathrm{n} \cdot{ }^{\circ} / \text { total }\right): \\
\text { - Tipo: }\end{array}$ \\
\hline
\end{tabular}


M. M. Ibáñez Ibáñez, M. C. Romero López, M. P. Jiménez Tejada

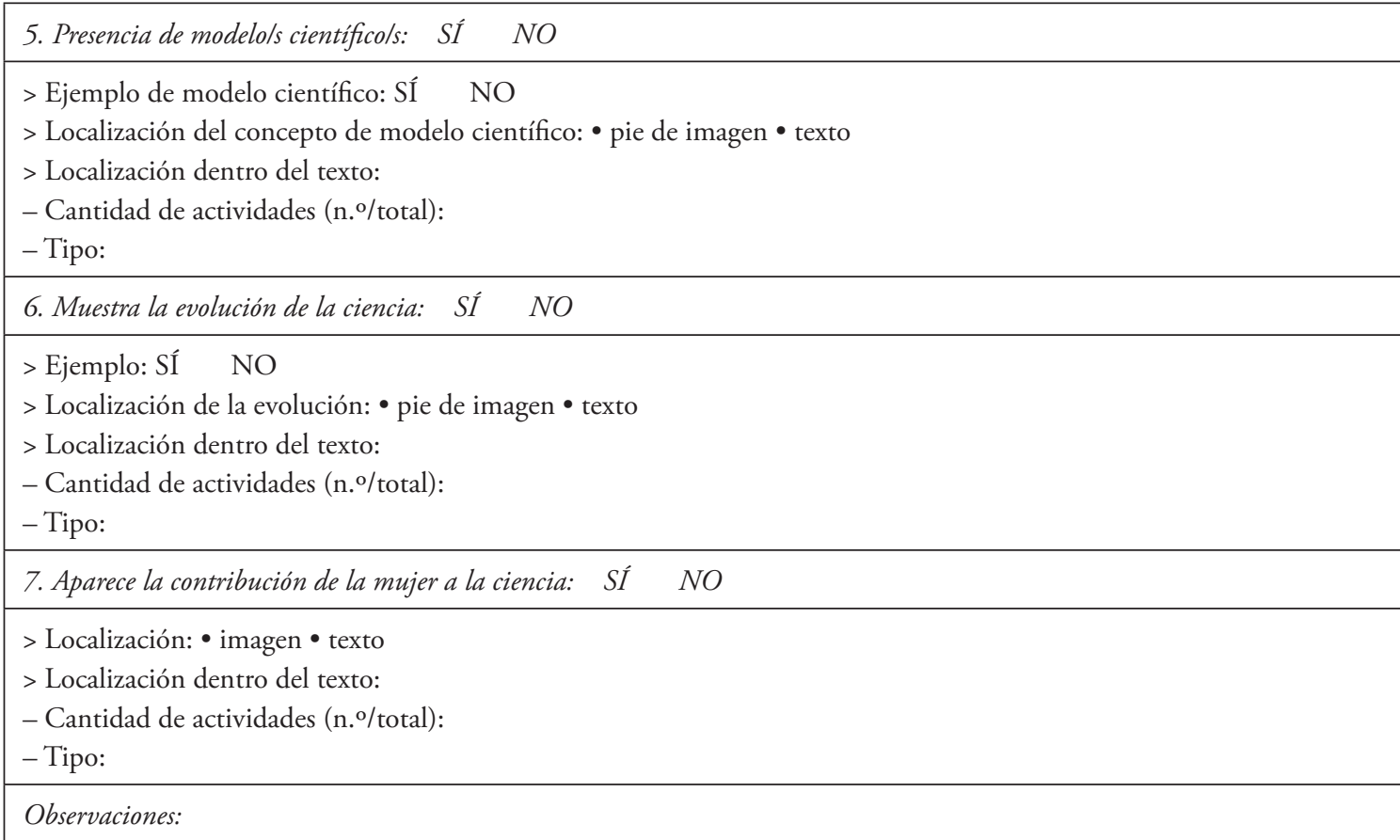




\title{
Which type of science is presented in Secondary Education textbooks?
}

\author{
Margarita Minerva Ibáñez Ibáñez, María del Carmen Romero López, María del Pilar Jiménez Tejada \\ Departamento de Didáctica de las Ciencias Experimentales. Universidad de Granada, España. \\ margaritaibanezibanez@gmail.com,romero@ugr.es, pjtejada@ugr.es
}

This paper presents an analysis of the introduction to science as made in biology and geology (BG) and physics and chemistry (PC) Secondary Education lessons as well as in their corresponding textbooks.

What science is, how it works, how scientists work and how science and society are related, is what we know today as the nature of science (NoS) (Clough, 2006; McComas et al., 1998). Naive views on science are commonly held (Afonso and Gilbert, 2010; Koskal, Cakiroglu and Geban, 2013) and are often reflected in textbooks (McComas, 1998). Therefore, a good reason to include NoS in education syllabuses would be to fight these misconceptions among society. The overall aim of this study is to characterise the view of NoS which is promoted in the textbooks which are frequently used in classrooms. In this sense, these following specific objectives are addressed: to determine whether the definitions given are consistent with the current view of NoS; to examine whether science and pseudoscience are differentiated; to detect the presence/absence of scientific models; to see whether the evolution of scientific thought is presented; and to observe whether women's contribution to science is acknowledged.

For this purpose, 31 textbooks (15 for BG and 16 for PC) were studied. The data were analysed through a mixed qualitative-quantitative approach. An initial examination showed that 11 books had no introductory science lesson. For the sake of consistency, and to address all the study goals, these 11 were excluded, and so the final sample analysed was made of 20 textbooks (5 for BG and 15 for PC).

Various deficiencies were found in these textbooks. Thus, in the introductory science lesson, $25 \%$ of the textbooks have neither a definition of science nor a description. Moreover, although science and pseudoscience are contrasted in $50 \%$ of the textbooks, this confrontation is not always consistent. Scientific methodology is described in $95 \%$ of the cases examined, but this description is frequently accompanied by misconceptions such as «the scientific method is a single and unique method» or "experimentation is the main activity of science». Only $35 \%$ of the textbooks refer to scientific models as a result of scientific work. Finally, the evolution of science and the contribution made by women are rarely addressed in detail.

With regards to the activities recommended to be performed in science classes, 455 of such activities were analysed, in two ways: the syllabus content worked and the activity type. Every activity can potentially be classified in more than one category. For the former of these aspects, the results obtained were consistent with what was found at the theoretical level. A scientific methodology is applied in 59,1\% of the activities, but the remaining aspects receive little attention. Regarding the types of activities, the following categories and results were observed: reproductive $(27,9 \%)$, deductive $(23,5 \%)$, applied $(22,0 \%)$, practical-experimental $(3,4 \%)$, logical-reflexive (20,4\%), critical-argumentative (2,2\%), bibliographic research (17,4\%), other $(5,0 \%)$.

The activities involving the student in practical or research work were considered separately. This kind of activity usually takes place at the end of the lessons, and so they are known as "final sections». In none of these sections is there any relation with the theoretical concepts analysed, and so these sections were not taken into account for the first aspect of the study. Each one was analysed as a single activity, hence 22 "final sections» presented in 13 textbooks were analysed. As expected from the nature of this kind of activity, applied (86\%) and practical-experimental activities (68 \%) were the most common types.

In view of the results obtained, we conclude that the introductory lessons analysed present a lack of reflection on what science is and how it works.

Textbooks usually reflect the official curriculum and are commonly used in the classroom. Hence, they are an essential part of teaching and learning, which makes them an important object of study and reflection in didactic research. 
